\title{
Ginzburg-Landau-Wilson Hamiltonian for a multi-component continuous system: a microscopic description
}

\author{
O.V.Patsahan \\ Institute for Condensed Matter Physics \\ of the National Academy of Sciences of Ukraine, \\ 1 Svientsitskii Str., 79011 Lviv, Ukraine
}

Received April 13, 2002

\begin{abstract}
Recently we proposed the microscopic approach to the description of the phase behaviour and critical phenomena in binary fluid mixtures. It was based on the method of collective variables (CV) with a reference system. The approach allowed us to obtain the functional of the Ginzburg-LandauWilson (GLW) Hamiltonian expressed in terms of the collective variables ("density" variables). The corresponding set of collective variables included the variable connected with the order parameter. In this paper, based on the previous results, we construct the GLW Hamiltonian in the phase space of the "field" variables $\hat{\phi}_{\vec{k}}$ (fluctuating fields) conjugate to the "density" variables. We apply the obtained GLW functional to the study of both the binary symmetrical mixture and the restricted primitive model. In the former case we consider the Gaussian approximation only and show that the obtained results are the same as those found previously using the CV method. In the latter case we calculate the phase diagram taking into account the powers of $\hat{\phi}_{\vec{k}}$ higher than the second one.
\end{abstract}

Key words: phase transition, a two-component continuous system, order parameter, fluctuating field

PACS: 05.70.Fh, 05.70.Jk, 65.10.+h

\section{Introduction}

Nowadays the theory of phase transitions and critical phenomena is well developed in general. It enables us to obtain both universal and non-universal properties for many model systems. However, a number of questions still remains open, for instance, a criticality of ionic fluids, a Yang-Yang anomaly and others. In order to answer these questions it is important to obtain the explicit form of an effective Ginzburg-Landau-Wilson (GLW) Hamiltonian with coefficients related to the microscopic parameters of the system. While for lattice models this task does not pose 
special difficulties, the case of continuous systems is more complicated from a mathematical point of view. Particularly, the latter case was studied in [1-5]. However, these considerations either did not go beyond the mean field approximation $[2,3]$ or just took into account Gaussian fluctuations $[1,4,5]$.

Another microscopic approach to the study of phase transitions was proposed in the late 80ies. First it was applied to a 3D Ising model [6] and then it was developed for a simple fluid near the gas-liquid critical point [7-9]. This theory has its origin in the approach based on a functional representation of a partition function in the collective variables (CV) space [10,11]. Its particular feature is a choice of the phase space in which the system is considered. Among the independent variables of this space there should be the ones connected with the order parameters. This phase space is formed by a set of CV. Each of them is a mode of density fluctuations corresponding to the specificity of the model under consideration. In particular, for a magnetic system, the CVs are the variables connected with spin density fluctuation modes, while for a one-component fluid they are connected with particle density fluctuation modes. This approach allows one to determine, on microscopic grounds, the explicit form of the effective GLW Hamiltonian and then to integrate the partition function in the neighbourhood of the phase transition point taking into account the renormalization group symmetry. As a result, non-classical critical exponents and analytical expressions for thermodynamic functions are obtained [6,9]. More recently this theory has been developed for a binary fluid mixture [12-17].

In contrast to lattice systems, the description of phase transitions in continuous systems has a number of the important peculiarities. On the one hand, as one usually does in the liquid state theory, we should distinguish a reference system (RS) describing the behaviour at short distances. This will allow us to take into consideration the short-range and the long-range interactions simultaneously. On the other hand, the grand canonical ensemble (GCE) should be used in order to describe the processes relating to the phase transitions in multi-component fluids in which the composition fluctuations play a crucial role (e.g., the gas-gas and liquid-liquid equilibria in binary fluid mixtures). The task of the development of the CV method for the case of the GCE is also caused by the problem of selecting the CV phase space which includes the variable connected with the order parameter.

The set of CV can be called "density" variables. The proper choice of the CV phase space is very important for determining the order parameter appearing in complex systems below the phase transition point. When the "density" variable connected with the order parameter is known we can represent the GLW Hamiltonian in the phase space of the "field" variables (fluctuating fields) conjugate to the "density" ones. This is the main purpose of the present paper. In section 2 using the Hubburd-Schofield method [18] we obtain the functional representation of a grand partition function of a multi-component continuous system in the phase space of "field" variables. A two-component system is considered in section 3. The fields conjugate to the CV connected with the order parameters are determined here. The case of the binary symmetrical fluid is discussed. In section 4 we use this approach to the study of a restricted primitive model (RPM). The terms of the order higher 
than the second one in the GLW Hamiltonian are taken into account. As a result, the phase diagram is obtained which demonstrates both the gas-liquid and charge ordering phase transitions. Section 5 contains some concluding remarks.

\section{Functional representation of a grand partition function of a multi-component continuous system}

Let us consider a classical $m$-component continuous system of interacting particles consisting of $N_{a_{1}}$ particles of species $a_{1}, N_{a_{2}}$ particles of species $a_{2}, \ldots$ and $N_{a_{m}}$ particles of species $a_{m}$. The system is in volume $V$ at temperature $T$.

The interaction potential between particles has a pairwise additive character and may be presented as a sum of two terms:

$$
U_{\gamma \delta}(r)=\psi_{\gamma \delta}(r)+\Phi_{\gamma \delta}(r),
$$

where $\psi_{\gamma \delta}(r)$ is a potential of a short-range repulsion that characterizes the mutual impermeability of particles. In the simplest case it can be chosen as an interaction between the two hard spheres $\sigma_{\gamma \gamma}$ and $\sigma_{\delta \delta} . \Phi_{\gamma \delta}(r)$ is an attractive part of the potential which dominates at large distances.

Let us start with a grand partition function

$$
\Xi=\sum_{N_{a_{1}}=0}^{\infty} \sum_{N_{a_{2}}=0}^{\infty} \ldots \sum_{N_{a_{m}}=0}^{\infty} \prod_{\gamma=a_{1}}^{a_{m}} \frac{z_{\gamma}^{N_{\gamma}}}{N_{\gamma} !} \int(\mathrm{d} \gamma) \exp \left[-\frac{\beta}{2} \sum_{\gamma \delta} \sum_{i j} U_{\gamma \delta}\left(r_{i j}\right)\right]
$$

where $(\mathrm{d} \Gamma)=\prod_{\gamma} \mathrm{d} \Gamma_{N_{\gamma}}, \mathrm{d} \Gamma_{N_{\gamma}}=\mathrm{d} \vec{r}_{1}^{\gamma} \mathrm{d} \vec{r}_{2}^{\gamma} \ldots \mathrm{d} \vec{r}_{N_{\gamma}}^{\gamma}$ is an element of the configurational space of the $\gamma$ th species; $z_{\gamma}$ is the fugacity of the $\gamma$ th species: $z_{\gamma}=\exp \left(\beta \mu_{\gamma}^{\prime}\right)$, $\mu_{\gamma}^{\prime}=\mu_{\gamma}+\beta^{-1} \ln \left[\left(2 \pi m_{\gamma} \beta^{-1}\right)^{3 / 2} / h^{3}\right] ; \beta=1 / k_{\mathrm{B}} T, k_{\mathrm{B}}$ is the Boltzmann constant, $T$ is temperature; $m_{\gamma}$ is mass of the $\gamma$ th species, $h$ is the Planck constant. $\mu_{\gamma}^{\prime}$ is determined from

$$
\frac{\partial \ln \Xi}{\partial \beta \mu_{\gamma}^{\prime}}=\left\langle N_{\gamma}\right\rangle,
$$

where $\left\langle N_{\gamma}\right\rangle$ is the average number of the $\gamma$ th species.

Let us introduce operators $\hat{\rho}_{\vec{k}, \gamma}$

$$
\hat{\rho}_{\vec{k}, \gamma}=\sum_{j=1}^{N_{\gamma}} \exp \left(-\mathrm{i} \vec{k} \vec{r}_{j}^{\gamma}\right),
$$

which are Fourier transforms of the particle number density operators. Now we write the attractive part of the potential in the form

$$
\sum_{i \neq j} \Phi_{\gamma \delta}\left(r_{i j}\right)=\frac{1}{V} \sum_{\vec{k}} \tilde{\Phi}_{\gamma \delta}(k)\left(\hat{\rho}_{\vec{k}, \gamma} \hat{\rho}_{-\vec{k}, \delta}-\hat{\rho}_{0, \gamma} \delta_{\gamma \delta}\right)
$$

where $\tilde{\Phi}_{\gamma \delta}(k)$ is the Fourier transform of $\Phi_{\gamma \delta}\left(r_{i j}\right)$ Then we can represent $\Xi$ as

$$
\Xi=\Xi_{\mathrm{RS}} \Xi_{1} .
$$


Here $\Xi_{\mathrm{RS}}$ is the grand partition function of the RS

$$
\Xi_{\mathrm{RS}}=\sum_{N_{a_{1}}=0}^{\infty} \sum_{N_{a_{2}}=0}^{\infty} \ldots \sum_{N_{a_{m}}=0}^{\infty} \prod_{\gamma=a_{1}}^{a_{m}} \frac{\exp \left(\beta \mu_{\gamma}^{(0)} N_{\gamma}\right)}{N_{\gamma} !} \int(\mathrm{d} \Gamma) \exp \left[-\frac{\beta}{2} \sum_{\gamma \delta} \sum_{i j} \psi_{\gamma \delta}\left(r_{i j}\right)\right]
$$

where $\mu_{\gamma}^{(0)}$ is the chemical potential of the $\gamma$ th species in the RS. $\Xi_{1}$ is as follows [19]:

$$
\Xi_{1}=\left\langle\exp \left\{\beta \sum_{\gamma=1}^{m} \mu_{\gamma}^{(1)} \hat{\rho}_{0, \gamma}-\frac{\beta}{2 V} \sum_{\gamma, \delta} \sum_{\vec{k}} \tilde{\Phi}_{\gamma \delta}(k) \hat{\rho}_{\vec{k}, \gamma} \hat{\rho}_{-\vec{k}, \delta}\right\}\right\rangle_{\mathrm{RS}} .
$$

Here $\langle\ldots\rangle_{\mathrm{RS}}$ means the average over RS. The chemical potential $\mu_{\gamma}^{(1)}=\mu_{\gamma}^{\prime}-\mu_{\gamma}^{(0)}$ is determined from the equation

$$
\frac{\partial \ln \Xi_{1}}{\partial \beta \mu_{\gamma}^{(1)}}=\left\langle N_{\gamma}\right\rangle
$$

We assume the thermodynamic and structural properties of the RS to be known.

In the matrix representation (2.2)-(2.4) can be written in the form

$$
\Xi=\Xi_{\mathrm{RS}}\left\langle\exp \left\{\beta \hat{\mu}^{(1)} \hat{\rho}_{0}+\frac{1}{2} \sum_{\vec{k}} \hat{\alpha}(k) \hat{\rho}_{\vec{k}} \hat{\rho}_{-\vec{k}}\right\}\right\rangle_{\mathrm{RS}}
$$

where $\hat{\rho}_{\vec{k}}$ denotes a column-vector

$$
\hat{\rho}_{\vec{k}}=\left(\begin{array}{c}
\hat{\rho}_{\vec{k}, 1} \\
\hat{\rho}_{\vec{k}, 2} \\
\vdots \\
\hat{\rho}_{\vec{k}, m}
\end{array}\right)
$$

$\hat{\mu}^{(1)}$ denotes a row-vector

$$
\hat{\mu}^{(1)}=\left(\mu_{1}^{(1)}, \mu_{2}^{(1)}, \ldots, \mu_{m}^{(1)}\right)
$$

and $\hat{\alpha}(k)$ is a symmetrical $(m \times m)$ matrix with elements $\alpha_{\gamma \delta}(k)=-(\beta / V) \tilde{\Phi}_{\gamma \delta}(k)$.

We perform in (2.5) the Hubburd-Stratonovich transformation [20]

$$
\int_{-\infty}^{\infty} \prod_{i=1}^{N} \mathrm{~d} x_{i} \exp \left(-\frac{1}{4} x_{i} V_{i j}^{-1} x_{j}+y_{i} x_{i}\right)=\text { const } \times \exp \left(y_{i} V_{i j} y_{j}\right),
$$

where summation over repeated indices is implied, and $\mathrm{V}$ is any symmetric positive matrix. As a result, we obtain

$$
\begin{aligned}
\Xi= & \Xi_{\mathrm{RS}}\left\langle\prod_{\vec{k}}(2 \pi)^{-1 / 2}(\operatorname{det} \hat{B}(k))^{1 / 2} \int_{-\infty}^{\infty} \ldots \int_{-\infty}^{\infty} \prod_{\gamma=1}^{m} \prod_{\vec{k}} \mathrm{~d} \hat{\phi}_{\vec{k}, \gamma}\right. \\
& \left.\times \exp \left\{-\frac{1}{2} \sum_{\vec{k}} \hat{B}(k) \hat{\phi}_{\vec{k}} \hat{\phi}_{-\vec{k}}+\sum_{\vec{k}} \hat{\rho}_{\vec{k}}\left(\hat{\phi}_{\vec{k}}+\delta_{\vec{k}} \beta \hat{\mu}^{(1)}\right)\right\}\right\rangle_{\mathrm{RS}},
\end{aligned}
$$


where $\hat{\phi}_{\vec{k}}$ is a row-vector with elements $\hat{\phi}_{\vec{k}, \gamma} \cdot \hat{B}(k)$ denotes an inverse matrix to $\hat{\alpha}(k)$ with elements $b_{i j}$.

After transformation $\hat{\phi}_{\vec{k}}^{\prime}=\hat{\phi}_{\vec{k}}+\delta_{\vec{k}} \beta \hat{\mu}^{(1)}$ in (2.7) we obtain

$$
\begin{gathered}
\Xi=\Xi_{\mathrm{RS}} \prod_{\vec{k}}(2 \pi)^{-1 / 2}(\operatorname{det} \hat{B}(k))^{1 / 2} \int_{-\infty}^{\infty} \ldots \int_{-\infty}^{\infty} \prod_{m=1}^{s} \prod_{\vec{k}} \mathrm{~d} \hat{\phi}_{\vec{k}, \gamma}^{\prime} \\
\times \exp \left\{-\frac{1}{2} \sum_{\vec{k}} \hat{B}(k) \hat{\phi}_{\vec{k}}^{\prime} \hat{\phi}_{-\vec{k}}^{\prime}+\beta \hat{\mu}^{(1)} \hat{B}(0) \hat{\phi}_{0}^{\prime}\right. \\
\left.-\frac{1}{2} \hat{B}(0)\left(\beta \hat{\mu}^{(1)}\right)^{2}\right\}\left\langle\exp \sum_{\vec{k}} \hat{\rho}_{\vec{k}} \hat{\phi}_{\vec{k}}^{\prime}\right\rangle_{\mathrm{RS}} .
\end{gathered}
$$

Here $\hat{\phi}_{\vec{k}}^{\prime}$ has a meaning of a "field" variable conjugate to a "density" variable $\hat{\rho}_{\vec{k}}$.

We can present the expression $\left\langle\exp \sum_{\vec{k}} \hat{\rho}_{\vec{k}} \hat{\phi}_{\vec{k}}^{\prime}\right\rangle_{\mathrm{RS}}$ in the form of a cumulant expansion $[21,22]$ :

$$
\left\langle\exp \sum_{\vec{k}} \hat{\rho}_{\vec{k}} \hat{\phi}_{\vec{k}}^{\prime}\right\rangle_{\mathrm{RS}}=\exp \sum_{n \geqslant 1} \hat{D}_{n}\left(\hat{\phi}^{\prime}\right)
$$

where

$$
\hat{D}_{n}\left(\hat{\phi}^{\prime}\right)=\frac{1}{n !} \sum_{\vec{k}_{1}, \ldots, \vec{k}_{n}} \hat{\mathfrak{M}}_{n}\left(\vec{k}_{1}, \ldots, \vec{k}_{n}\right) \hat{\phi}_{\vec{k}_{1}}^{\prime} \hat{\phi}_{\vec{k}_{2}}^{\prime} \ldots \hat{\phi}_{\vec{k}_{n}}^{\prime},
$$

$\hat{\mathfrak{M}}_{n}\left(\vec{k}_{1}, \ldots, \vec{k}_{n}\right)$ is a symmetrical $\underbrace{m \times m \times \ldots \times m}_{n}$ matrix (see Appendix A).

In general, the dependence of $\mathfrak{M}_{n}^{\gamma_{1} \ldots \gamma_{n}}\left(\vec{k}_{1}, \ldots, \vec{k}_{n}\right)$ on wave vectors $\vec{k}_{1}, \ldots, \vec{k}_{n}$ is complicated. Since we are interested in the critical properties, the small $\vec{k}$ expansion of the cumulants can be considered. Hereafter we shall replace $\mathfrak{M}_{n}^{\gamma_{1} \ldots \gamma_{n}}\left(\vec{k}_{1}, \ldots, \vec{k}_{n}\right)$ by their values in the long-wavelength limit $\mathfrak{M}_{n}^{\gamma_{1} \ldots \gamma_{n}}(0, \ldots, 0)$. The recurrence formulas for $\mathfrak{M}_{n}^{\gamma_{1} \ldots \gamma_{n}}(0, \ldots, 0)$ are obtained in [23].

As a result, we can present (2.8) in the form

$$
\begin{aligned}
\Xi=\Xi_{\mathrm{RS}} \prod_{\vec{k}}(2 \pi)^{-1 / 2}(\operatorname{det} \hat{B}(k))^{1 / 2} \int_{-\infty}^{\infty} \ldots \int_{-\infty}^{\infty} \prod_{m=1}^{s} \prod_{\vec{k}} \mathrm{~d} \hat{\phi}_{\vec{k}, \gamma}^{\prime} \\
\times \exp \left\{-\frac{1}{2} \sum_{\vec{k}}\left(\hat{B}(k)-\hat{\mathfrak{M}}_{2}(0,0)\right) \hat{\phi}_{\vec{k}}^{\prime} \hat{\phi}_{-\vec{k}}^{\prime}+\left(\beta \hat{\mu}^{(1)} \hat{B}(0)+\hat{\mathfrak{M}}_{1}(0)\right) \hat{\phi}_{0}^{\prime}\right. \\
\left.-\frac{1}{2} \hat{B}(0)\left(\beta \hat{\mu}^{(1)}\right)^{2}\right\} \exp \sum_{n \geqslant 3} \hat{D}_{n}\left(\hat{\phi}^{\prime}\right)
\end{aligned}
$$

\section{A two-component system}

Let us consider a classical two-component system of interacting particles consisting of $N_{a}$ particles of $a$ species and $N_{b}$ particles of $b$ species. We pass to new variables $\hat{\phi}_{\vec{k}}^{+}$and $\hat{\phi}_{\vec{k}}^{-}$in (2.8)-(2.10) by means of the orthogonal linear transformation

$$
\hat{\phi}_{\vec{k}}^{+}=\frac{1}{\sqrt{2}}\left(\hat{\phi}_{\vec{k}, a}+\hat{\phi}_{\vec{k}, b}\right), \quad \hat{\phi}_{\vec{k}}^{-}=\frac{1}{\sqrt{2}}\left(\hat{\phi}_{\vec{k}, a}-\hat{\phi}_{\vec{k}, b}\right),
$$


As a result, we obtain for $\Xi$

$$
\begin{aligned}
\Xi=\Xi_{\mathrm{RS}} \prod_{\vec{k}}(2 \pi)^{-1 / 2}(\operatorname{det} \hat{B}(k))^{1 / 2} \int\left(\mathrm{d} \hat{\phi}^{+}\right)\left(\mathrm{d} \hat{\phi}^{-}\right) \\
\times \exp \left\{-\frac{1}{2} \sum_{\gamma, \delta} b_{\gamma \delta} \beta \mu_{\gamma}^{(1)} \beta \mu_{\delta}^{(1)}+M^{+} \hat{\phi}_{0}^{+}+M^{-} \hat{\phi}_{0}^{-}\right. \\
\quad-\frac{1}{2} \sum_{\vec{k}}\left[\tilde{\mathcal{V}}(k) \hat{\phi}_{\vec{k}}^{+} \hat{\phi}_{-\vec{k}}^{+}+2 \tilde{\mathcal{U}}(k) \hat{\phi}_{\vec{k}}^{+} \hat{\phi}_{-\vec{k}}^{-}+\tilde{\mathcal{W}}(k) \hat{\phi}_{\vec{k}}^{-} \hat{\phi}_{-\vec{k}}^{-}\right] \\
\left.\left.+\sum_{n \geqslant 1} \hat{D}_{n}\left(\hat{\phi}^{+}, \hat{\phi}^{-}\right)\right]\right\} .
\end{aligned}
$$

Here the following notations are introduced:

$$
\begin{aligned}
& M^{+}=\frac{1}{\sqrt{2}}\left[\beta \mu_{a}^{(1)}(\tilde{\mathcal{V}}(0)+\tilde{\mathcal{U}}(0))+\beta \mu_{b}^{(1)}(\tilde{\mathcal{V}}(0)-\tilde{\mathcal{U}}(0))\right], \\
& M^{-}=\frac{1}{\sqrt{2}}\left[\beta \mu_{a}^{(1)}(\tilde{\mathcal{W}}(0)+\tilde{\mathcal{U}}(0))-\beta \mu_{b}^{(1)}(\tilde{\mathcal{W}}(0)-\tilde{\mathcal{U}}(0))\right] \\
& \tilde{\mathcal{V}}(k)=\left(b_{11}(k)+b_{22}(k)+2 b_{12}(k)\right) / 2, \\
& \tilde{\mathcal{W}}(k)=\left(b_{11}(k)+b_{22}(k)-2 b_{12}(k)\right) / 2 \text {, } \\
& \tilde{\mathcal{U}}(k)=\left(b_{11}(k)-b_{22}(k)\right) / 2 \text {, } \\
& \hat{D}_{1}\left(\hat{\phi}^{+}, \hat{\phi}^{-}\right)=\frac{1}{\sqrt{2}}\left(\mathfrak{M}_{1}^{(0)} \hat{\phi}_{0}^{+}+\mathfrak{M}_{1}^{(1)} \hat{\phi}_{0}^{-}\right), \\
& \hat{D}_{2}\left(\hat{\phi}^{+}, \hat{\phi}^{-}\right)=\frac{1}{2 !} \sum_{\vec{k}_{1}, \vec{k}_{2}} \frac{1}{(\sqrt{2})^{2}}\left(\mathfrak{M}_{2}^{(0)} \hat{\phi}_{\vec{k}_{1}}^{+} \hat{\phi}_{\vec{k}_{2}}^{+}+2 \mathfrak{M}_{2}^{(1)} \hat{\phi}_{\vec{k}_{1}}^{+} \hat{\phi}_{\vec{k}_{2}}^{-}\right. \\
& \left.+\mathfrak{M}_{2}^{(2)} \hat{\phi}_{\vec{k}_{1}}^{-} \hat{\phi}_{\vec{k}_{2}}^{-}\right) \delta_{\vec{k}_{1}+\vec{k}_{2}} \\
& \hat{D}_{3}\left(\hat{\phi}^{+}, \hat{\phi}^{-}\right)=\frac{1}{3 !} \sum_{\vec{k}_{1} \ldots \vec{k}_{3}} \frac{1}{(\sqrt{2})^{3}}\left(\mathfrak{M}_{3}^{(0)} \hat{\phi}_{\vec{k}_{1}}^{+} \hat{\phi}_{\vec{k}_{2}}^{+} \hat{\phi}_{\vec{k}_{3}}^{+}+3 \mathfrak{M}_{3}^{(1)} \hat{\phi}_{\vec{k}_{1}}^{+} \hat{\phi}_{\vec{k}_{2}}^{+} \hat{\phi}_{\vec{k}_{3}}^{-}\right. \\
& \left.+3 \mathfrak{M}_{3}^{(2)} \hat{\phi}_{\vec{k}_{1}}^{+} \hat{\phi}_{\vec{k}_{2}}^{-} \hat{\phi}_{\vec{k}_{3}}^{-}+\mathfrak{M}_{3}^{(3)} \hat{\phi}_{\vec{k}_{1}}^{-} \hat{\phi}_{\vec{k}_{2}}^{-} \hat{\phi}_{\vec{k}_{3}}^{-}\right) \delta_{\vec{k}_{1}+\vec{k}_{2}+\vec{k}_{3}}, \\
& \hat{D}_{4}\left(\hat{\phi}^{+}, \hat{\phi}^{-}\right)=\frac{1}{4 !} \sum_{\vec{k}_{1} \ldots \vec{k}_{4}} \frac{1}{(\sqrt{2})^{4}}\left(\mathfrak{M}_{4}^{(0)} \hat{\phi}_{\vec{k}_{1}}^{+} \hat{\phi}_{\vec{k}_{2}}^{+} \hat{\phi}_{\vec{k}_{3}}^{+} \phi_{\vec{k}_{4}}^{+}+4 \mathfrak{M}_{4}^{(1)} \hat{\phi}_{\vec{k}_{1}}^{+} \hat{\phi}_{\vec{k}_{2}}^{+} \hat{\phi}_{\vec{k}_{3}}^{+} \hat{\phi}_{\vec{k}_{4}}^{-}\right. \\
& +6 \mathfrak{M}_{4}^{(2)} \hat{\phi}_{\vec{k}_{1}}^{+} \hat{\phi}_{\vec{k}_{2}}^{+} \hat{\phi}_{\vec{k}_{3}}^{-} \hat{\phi}_{\vec{k}_{4}}^{-}+4 \mathfrak{M}_{4}^{(3)} \hat{\phi}_{\vec{k}_{1}}^{+} \hat{\phi}_{\vec{k}_{2}}^{-} \hat{\phi}_{\vec{k}_{3}}^{-} \hat{\phi}_{\vec{k}_{4}}^{-} \\
& \left.+\mathfrak{M}_{4}^{(4)} \hat{\phi}_{\vec{k}_{1}}^{-} \hat{\phi}_{\vec{k}_{2}}^{-} \hat{\phi}_{\vec{k}_{3}}^{-} \hat{\phi}_{\vec{k}_{4}}^{-}\right) \delta_{\vec{k}_{1}+\vec{k}_{2}+\vec{k}_{3}+\vec{k}_{4}} \text {. }
\end{aligned}
$$

The expressions for $\mathfrak{M}_{n}^{\left(i_{n}\right)}$ are the same as those in [14].

Let us consider the Gaussian approximation of the functional of the grand partition function $(n \leqslant 2$ in $(3.1))$ :

$$
\Xi=\Xi_{\mathrm{RS}} \prod_{\vec{k}}(2 \pi)^{-1 / 2}(\operatorname{det} \hat{B}(k))^{1 / 2} \int\left(\mathrm{d} \hat{\phi}^{+}\right)\left(\mathrm{d} \hat{\phi}^{-}\right)
$$




$$
\begin{aligned}
\times \exp \{ & -\frac{1}{2} \sum_{\gamma, \delta} \beta \mu_{\gamma}^{(1)} b_{\gamma \delta} \beta \mu_{\delta}^{(1)}+\left(M^{+}+\overline{\mathfrak{M}}_{1}^{(0)}\right) \hat{\phi}_{0}^{+}+\left(M^{-}+\overline{\mathfrak{M}}_{1}^{(1)}\right) \hat{\phi}_{0}^{-} \\
& \left.-\frac{1}{2} \sum_{\vec{k}}\left[A_{11}(k) \hat{\phi}_{\vec{k}}^{+} \hat{\phi}_{-\vec{k}}^{+}+2 A_{12}(k) \hat{\phi}_{\vec{k}}^{+} \hat{\phi}_{-\vec{k}}^{-}+A_{22}(k) \hat{\phi}_{\vec{k}}^{-} \hat{\phi}_{-\vec{k}}^{-}\right]\right\}
\end{aligned}
$$

where

$$
\begin{aligned}
A_{11}(k) & =\tilde{\mathcal{V}}(k)-\overline{\mathfrak{M}}_{2}^{(0)}, \\
A_{22}(k) & =\tilde{\mathcal{W}}(k)-\overline{\mathfrak{M}}_{2}^{(2)}, \\
A_{12}(k) & =\tilde{\mathcal{U}}(k)-\overline{\mathfrak{M}}_{2}^{(1)}, \\
\overline{\mathfrak{M}}_{n}^{\left(i_{n}\right)} & =\frac{1}{\sqrt{2}^{n}} \mathfrak{M}_{n}^{\left(i_{n}\right)} .
\end{aligned}
$$

In order to determine the "field" variables conjugate to the order parameters we diagonalize the square form in (3.9) by means of the orthogonal transformation:

$$
\begin{aligned}
& \hat{\phi}_{\vec{k}}^{+}=\mathcal{A}(k) \chi_{1, \vec{k}}+\mathcal{B}(k) \chi_{2, \vec{k}} \\
& \hat{\phi}_{\vec{k}}^{-}=\mathcal{C}(k) \chi_{1, \vec{k}}+\mathcal{D}(k) \chi_{2, \vec{k}}
\end{aligned}
$$

where

$$
\begin{aligned}
& \mathcal{A}(k)=\frac{\beta_{1}}{\sqrt{1+\beta_{1}^{2}}}, \quad \mathcal{B}(k)=\frac{\beta_{2}}{\sqrt{1+\beta_{2}^{2}}}, \\
& \mathcal{C}(k)=\frac{1}{\sqrt{1+\beta_{1}^{2}}}, \quad \mathcal{D}(k)=\frac{1}{\sqrt{1+\beta_{2}^{2}}}, \\
& \beta_{1,2}=-\frac{A_{22}-A_{11} \mp \sqrt{\left(A_{11}-A_{22}\right)^{2}+4 A_{12}^{2}}}{2 A_{12}} .
\end{aligned}
$$

As a result, we get for the square form

$$
-\frac{1}{2} \sum_{s=1}^{2} \sum_{\vec{k}} \varepsilon_{s}(k) \chi_{s, \vec{k}} \chi_{s,-\vec{k}}
$$

where

$$
\varepsilon_{1,2}(k)=A_{11}(k)+A_{22}(k) \mp \sqrt{\left(A_{11}(k)-A_{22}(k)\right)^{2}+4 A_{12}^{2}(k)} .
$$

In a general case only one of the quantities $\varepsilon_{1}(k)$ and $\varepsilon_{2}(k)$ is a critical one, no matter whether the system approaches the gas-liquid or mixing-demixing critical point $[15,16]$. 
We pass in (3.5)-(3.8) to new variables $\chi_{s, \vec{k}}$ (see Appendix B). As a result, the functional of the grand partition function (3.9) has the form

$$
\begin{aligned}
\Xi=\Xi_{\mathrm{RS}} \prod_{\vec{k}}(2 \pi)^{-1 / 2}(\operatorname{det} \hat{B}(k))^{1 / 2} \int\left(\mathrm{d} \chi_{1}\right)\left(\mathrm{d} \chi_{2}\right) \\
\times \exp \left\{-\frac{1}{2} \sum_{\gamma, \delta} \beta \mu_{\gamma}^{(1)} b_{\gamma \delta} \beta \mu_{\delta}^{(1)}+\left(\mathcal{A} M^{+}+\mathcal{C} M^{-}\right) \chi_{1,0}+\left(\mathcal{B} M^{+}+\mathcal{D} M^{-}\right) \chi_{2,0}\right. \\
\left.\quad-\frac{1}{2} \sum_{s=1}^{2} \sum_{\vec{k}} \varepsilon_{s}(k) \chi_{s, \vec{k}} \chi_{s,-\vec{k}}+\sum_{n \geqslant 3} \hat{D}_{n}\left(\chi_{1}, \chi_{2}\right)\right\}
\end{aligned}
$$

In order to obtain the effective GLW Hamiltonian we can follow the program proposed in [13,16], namely: (1) to determine the critical branch $\varepsilon_{s}(k)$ and the ordering fields $\chi_{s, \vec{k}}$ connected to it; (2) to integrate over the remaining $\chi_{s, \vec{k}}$ (irrelevant variables), using the Gaussian density measure as the basic one; (3) as a result of the integration performed in (2), to construct the functional including higher powers of the ordering fields $\chi_{s, \vec{k}}$ than the second power. As a result, we obtain the GLW Hamiltonian with coefficients which are the known functions of the microscopic parameters, temperature, concentration and density.

A symmetrical mixture. We consider a symmetrical binary fluid mixture (SBFM), i.e. a system in which the two pure components "a" and "b" are identical and only interactions between the particles of dissimilar species differ: $\sigma_{a a}=\sigma_{b b}=\sigma$ and $\Phi_{a a}(r)=\Phi_{b b}(r)=\Phi(r) \neq \Phi_{a b}(r)$. Notwithstanding its simplicity, the SBFM exhibits all the three types of two-phase equilibria which are observed in real binary fluids, namely: gas-liquid, liquid-liquid and gas-gas equilibria. In this case the functional of the grand partition function (3.13) is reduced to the form

$$
\begin{aligned}
\Xi=\Xi_{\mathrm{RS}} \prod_{\vec{k}}(2 \pi)^{-1 / 2}(\operatorname{det} \hat{B}(k))^{1 / 2} \int\left(\mathrm{d} \hat{\phi}^{+}\right)\left(\mathrm{d} \hat{\phi}^{-}\right) \\
\times \exp \left\{-\frac{1}{2} \sum_{\gamma, \delta} \beta \mu_{\gamma}^{(1)} b_{\gamma \delta} \beta \mu_{\delta}^{(1)}+M^{+} \hat{\phi}_{0}^{+}+M^{-} \hat{\phi}_{0}^{-}\right. \\
\left.\quad-\frac{1}{2} \sum_{\vec{k}}\left(\varepsilon_{1}(k) \hat{\phi}_{\vec{k}}^{-} \hat{\phi}_{-\vec{k}}^{-}+\varepsilon_{2}(k) \hat{\phi}_{\vec{k}}^{+} \hat{\phi}_{-\vec{k}}^{+}\right)+\sum_{n \geqslant 3} \hat{D}_{n}\left(\hat{\phi}^{+}, \hat{\phi}^{-}\right)\right\}
\end{aligned}
$$

where

$$
\begin{gathered}
\varepsilon_{1}(k)=\frac{1}{\alpha(k)-\alpha_{a b}(k)}-\overline{\mathfrak{M}}_{2}^{(2)}, \\
\varepsilon_{2}(k)=\frac{1}{\alpha(k)+\alpha_{a b}(k)}-\overline{\mathfrak{M}}_{2}^{(0)}, \\
M^{+}=\frac{1}{\sqrt{2}}\left(\langle N\rangle+\frac{2 \beta \mu_{a}^{(1)}}{\alpha(0)+\alpha_{a b}(0)}\right), \quad M^{-} \equiv 0
\end{gathered}
$$

and in (3.5)-(3.8) all cumulants $\overline{\mathfrak{M}}_{n}^{\left(i_{n}\right)} \equiv 0$ if $i_{n}$ are odd [13]. 
The $n$th cumulant $\mathfrak{M}_{n}^{\left(i_{n}\right)}$ with $i_{n}=0$ is connected with the $n$th structure factor of the one-component system $S_{n}(0)[13]$ :

$$
\mathfrak{M}_{n}^{(0)}(0)=\langle N\rangle S_{n}(0)
$$

Structure factors $S_{n}(0)$ with $n \geqslant 2$ can be obtained from $S_{2}(0)$ by means of a chain of equations for correlation functions [24]. Cumulants with $i_{n} \neq 0$ can be expressed in terms of $\mathfrak{M}_{n}^{(0)}(0)$ (see formulae (4.8) in [13]):

$$
\overline{\mathfrak{M}}_{n}^{(2)}=\frac{\overline{\mathfrak{M}}_{n-1}^{(0)}}{\sqrt{2}}, \quad \overline{\mathfrak{M}}_{n}^{(4)}=\frac{3}{2} \overline{\mathfrak{M}}_{n-2}^{(0)}-\frac{2}{\sqrt{2}} \overline{\mathfrak{M}}_{n-3}^{(0)} .
$$

As a result, we can rewrite (3.14)-(3.16) in the form

$$
\Xi=C \Xi_{\mathrm{RS}} \int\left(\mathrm{d} \hat{\phi}^{+}\right)\left(\mathrm{d} \hat{\phi}^{-}\right) \exp (-\beta \mathcal{H}),
$$

where

$$
C=\prod_{\vec{k}}(2 \pi)^{-1 / 2}(\operatorname{det} \hat{B}(k))^{1 / 2} \exp \left\{-\frac{1}{2} \sum_{\gamma, \delta} \beta \mu_{\gamma}^{(1)} b_{\gamma \delta} \beta \mu_{\delta}^{(1)}\right\} .
$$

$\mathcal{H}$ is the Hamiltonian expressed in terms of the two fluctuating fields $\hat{\phi}_{\vec{k}}^{+}$and $\hat{\phi}_{\vec{k}}^{-}$(in the approximation of the $\phi^{4}$ model):

$$
\begin{aligned}
\mathcal{H}= & -M^{+} \hat{\phi}_{0}^{+}-M^{-} \hat{\phi}_{0}^{-}+\frac{1}{2} \sum_{\vec{k}}\left(\varepsilon_{1}(k) \hat{\phi}_{\vec{k}}^{-} \hat{\phi}_{-\vec{k}}^{-}+\varepsilon_{2}(k) \hat{\phi}_{\vec{k}}^{+} \hat{\phi}_{-\vec{k}}^{+}\right) \\
& -\frac{1}{3 !} \sum_{\vec{k}_{1} \ldots \vec{k}_{3}}\left(\overline{\mathfrak{M}}_{3}^{(0)} \hat{\phi}_{\vec{k}_{1}}^{+} \hat{\phi}_{\vec{k}_{2}}^{+} \hat{\phi}_{\vec{k}_{3}}^{+}+3 \overline{\mathfrak{M}}_{3}^{(2)} \hat{\phi}_{\vec{k}_{1}}^{+} \hat{\phi}_{\vec{k}_{2}}^{-} \hat{\phi}_{\vec{k}_{3}}^{-}\right) \delta_{\vec{k}_{1}+\vec{k}_{2}+\vec{k}_{3}} \\
& -\frac{1}{4 !} \sum_{\vec{k}_{1} \ldots \vec{k}_{4}}\left(\overline{\mathfrak{M}}_{4}^{(0)} \hat{\phi}_{\vec{k}_{1}}^{+} \hat{\phi}_{\vec{k}_{2}}^{+} \hat{\phi}_{\vec{k}_{3}}^{+} \phi_{\vec{k}_{4}}^{+}+6 \overline{\mathfrak{M}}_{4}^{(2)} \hat{\phi}_{\vec{k}_{1}}^{+} \hat{\phi}_{\vec{k}_{2}}^{+} \hat{\phi}_{\vec{k}_{3}}^{-} \hat{\phi}_{\vec{k}_{4}}^{-}\right. \\
& \left.+\overline{\mathfrak{M}}_{4}^{(4)} \hat{\phi}_{\vec{k}_{1}}^{-} \hat{\phi}_{\vec{k}_{2}}^{-} \hat{\phi}_{\vec{k}_{3}}^{-} \hat{\phi}_{\vec{k}_{4}}^{-}\right) \delta_{\vec{k}_{1}+\vec{k}_{2}+\vec{k}_{3}+\vec{k}_{4}} .
\end{aligned}
$$

The fluctuating fields $\hat{\phi}_{\vec{k}}^{+}$and $\hat{\phi}_{\vec{k}}^{-}$are conjugate to the fluctuating (or collective) densities, namely: $\hat{\rho}_{\vec{k}}=\hat{\rho}_{\vec{k}, a}+\hat{\rho}_{\vec{k}, b}$ and $\hat{c}_{\vec{k}}=\hat{\rho}_{\vec{k}, a}-\hat{\rho}_{\vec{k}, b}$. Lately [14], for the SBFM we obtained the expression for the effective GLW Hamiltonian expressed in terms of the collective variables (fluctuating densities) $\rho_{\vec{k}}$ and $c_{\vec{k}}$. The two representations yield the same results in the Gaussian approximation (see [13]). As was shown, in such a system the two phase transitions can occur, namely, the gas-liquid and mixingdemixing phase transitions. The corresponding critical temperatures are determined from the equations (in the Gaussian approximation):

$$
\varepsilon_{1}(k=0)=0, \quad \varepsilon_{2}(k=0)=0 .
$$

In the above formulas an interaction in the system was not specified. The SBFM was studied in detail within the framework of the method of CV and the following model systems were considered: the hard-core Yukawa system [25], the hard-core Morse system [26] and the hard-core square-well system [14,17]. In the last case the non-universal critical properties were studied within the framework of the $\phi^{4}$ model. Below we shall use the approach proposed to the ionic fluid. 


\section{Restricted primitive model (RPM)}

We consider the simplest continuous model of an ionic fluid, namely, the restricted primitive model (RPM). It consists of $N=N_{+}+N_{-}$hard spheres of diameter $\sigma$ with $N_{+}$carrying charges $+q$ and $N_{-}\left(=N_{+}\right)$charges $-q$, in a medium of dielectric constant $D$. The interaction potential of the RPM has the form

$$
U_{\gamma \delta}(r)=\left\{\begin{array}{ll}
\infty, & r<\sigma \\
\frac{q_{\gamma} q_{\delta}}{D r}, & r \geqslant \sigma
\end{array} \quad, \quad q_{i}= \pm q\right.
$$

We split the potential $U_{\gamma \delta}(r)$ into short- and long-range parts using the WeeksChandler-Andersen partition [27]. As a result, we have

$$
\begin{aligned}
& \psi_{\gamma \delta}(r)=\left\{\begin{array}{ll}
\infty, & r \leqslant \sigma \\
0, & r>\sigma
\end{array},\right. \\
& \Phi_{\gamma \delta}(r)=\left\{\begin{array}{ll}
\frac{q_{\gamma} q_{\delta}}{D \sigma}, & r \leqslant \sigma \\
\frac{q_{\gamma} q_{\delta}}{D r}, & r>\sigma
\end{array} .\right.
\end{aligned}
$$

The Fourier transform of $\Phi_{\gamma \delta}(r)$ has the form

$$
\beta \rho \tilde{\Phi}_{\gamma \delta}(k)=24 \beta^{*} \eta \frac{\sin x}{x^{3}},
$$

where $\beta^{*}=\frac{\beta q^{2}}{D \sigma}, \eta=\frac{\pi}{6} \rho \sigma^{3}$ is fraction density, $x=k \sigma$.

Let us start with formulas (3.14)-(3.16). One must remember that for the RPM the transformation (2.6) has a formal character only. Thus the transition from the SBFM to the RPM should be performed in the final formulas for thermodynamic functions. For the RPM the fluctuating fields $\hat{\phi}_{\vec{k}}^{+}$and $\hat{\phi}_{\vec{k}}^{-}$are conjugate to the fluctuating densities $\hat{\rho}_{\vec{k}}^{+}$and $\hat{\rho}_{\vec{k}}^{-}$, the total number density and the charge density respectively. For this model the Gaussian approximation yields the only phase transition, connected with the charge ordering. The corresponding boundary of stability is determined from the condition $\varepsilon_{1}(k)=0$ or from the equation

$$
T^{*}=-24 \eta \frac{\sin x}{x^{3}}, \quad T^{*}=\frac{1}{\beta^{*}} .
$$

As a result, the equation of the charge ordering spinodal line is obtained

$$
T_{\mathrm{c}}^{*}\left(x^{*}, \eta\right)=-8 \eta \frac{\cos x^{*}}{x^{* 2}}
$$

where $x^{*}$ is determined from the condition

$$
\tan x^{*}=\frac{x^{*}}{3}
$$

which yields $x^{*} \simeq 4.0783$. 
In order to obtain the gas-liquid critical point we should take into consideration the order terms higher than the second order terms. As it was proposed in [13,16], we integrate in (3.17)-(3.18) (taking into account (3.15)-(3.16)) over $\hat{\phi}_{\vec{k}}^{-}$with the Gaussian measure density as the basic one.

We consider the integral

$$
\mathcal{I}=\int\left(\mathrm{d} \hat{\phi}^{-}\right) \exp \left(-\frac{1}{2} \sum_{\vec{k}} \tilde{\varepsilon}_{1}(k) \hat{\phi}_{\vec{k}}^{-} \hat{\phi}_{-\vec{k}}^{-}\right)\left(1+\frac{1}{2 !} \sum_{\vec{k}} \hat{\phi}_{\vec{k}}^{-} \hat{\phi}_{-\vec{k}}^{-} \mathbf{A}_{1}+\ldots\right),
$$

where

$$
\mathbf{A}_{1}=\overline{\mathfrak{M}}_{3}^{(2)} \hat{\phi}_{0}^{+}+\frac{1}{2} \overline{\mathfrak{M}}_{4}^{(2)} \sum_{\vec{k}_{1}} \hat{\phi}_{\overrightarrow{k_{1}}}^{+} \hat{\phi}_{-k_{1}}^{+}
$$

After the integration in (4.4)(taking into account the first term of the expansion) we obtain

$$
\Xi=C C^{\prime} \Xi_{\mathrm{RS}} \int\left(\mathrm{d} \hat{\phi}^{+}\right) \exp \left(-\beta \mathcal{H}^{\prime}\right)
$$

where the following notations are introduced

$$
\begin{aligned}
C^{\prime}= & \prod_{\vec{k}} \sqrt{\frac{2 \pi}{\tilde{\varepsilon}_{1}(k)}}, \\
\mathcal{H}^{\prime}= & -\tilde{M}^{+} \hat{\phi}_{0}^{+}+\frac{1}{2} \sum_{\vec{k}} \tilde{\varepsilon}_{2}(k) \hat{\phi}_{\vec{k}}^{+} \hat{\phi}_{-\vec{k}}^{+}-\frac{1}{3 !} \sum_{\vec{k}_{1} \ldots \vec{k}_{3}} \overline{\mathfrak{M}}_{3}^{(0)} \hat{\phi}_{\vec{k}_{1}}^{+} \hat{\phi}_{\vec{k}_{2}}^{+} \hat{\phi}_{\vec{k}_{3}}^{+} \delta_{\vec{k}_{1}+\vec{k}_{2}+\vec{k}_{3}} \\
& -\frac{1}{4 !} \sum_{\vec{k}_{1} \ldots \vec{k}_{4}} \overline{\mathfrak{M}}_{4}^{(0)} \hat{\phi}_{\vec{k}_{1}}^{+} \hat{\phi}_{\vec{k}_{2}}^{+} \hat{\phi}_{\vec{k}_{3}}^{+} \hat{\phi}_{\vec{k}_{4}}^{+} \delta_{\vec{k}_{1}+\vec{k}_{2}+\vec{k}_{3}+\vec{k}_{4}}, \\
\tilde{M}^{+}= & M^{+}+\overline{\mathfrak{M}}_{3}^{(2)} \tilde{a}(\beta) \\
\tilde{\varepsilon}_{2}(k)= & \varepsilon_{2}(k)-\overline{\mathfrak{M}}_{4}^{(2)} \tilde{a}(\beta)
\end{aligned}
$$

where

$$
\tilde{a}(\beta)=\frac{1}{2} \sum_{\vec{k}} \frac{1}{\varepsilon_{1}(k)} .
$$

Next, the shift is carried out in order to eliminate the cubic term in (4.7)

$$
\hat{\phi}_{\vec{k}}^{+}=\hat{\phi}_{\vec{k}}^{\prime+}+\Delta \delta_{\vec{k}},
$$

where $\Delta=-\overline{\mathfrak{M}}_{3}^{(0)} / \overline{\mathfrak{M}}_{4}^{(0)}$.

Then (4.5)-(4.7) has the form

$$
\Xi=C C^{\prime} C^{\prime \prime} \Xi_{\mathrm{RS}} \int\left(\mathrm{d} \hat{\phi}^{\prime+}\right) \exp (-\beta \overline{\mathcal{H}}),
$$

where

$$
C^{\prime \prime}=-\tilde{M}^{+} \frac{\overline{\mathfrak{M}}_{3}^{(0)}}{\overline{\mathfrak{M}}_{4}^{(0)}}-\frac{1}{2} \tilde{\varepsilon}_{2}\left(\frac{\overline{\mathfrak{M}}_{3}^{(0)}}{\overline{\mathfrak{M}}_{4}^{(0)}}\right)^{2}-\frac{1}{8} \frac{\left(\overline{\mathfrak{M}}_{3}^{(0)}\right)^{4}}{\left(\overline{\mathfrak{M}}_{4}^{(0)}\right)^{3}}
$$




$$
\begin{aligned}
\overline{\mathcal{H}}= & -\bar{M}^{+} \hat{\phi}_{0}^{+}+\frac{1}{2} \sum_{\vec{k}} \bar{\varepsilon}_{2}(k) \hat{\phi}_{\vec{k}}^{+} \hat{\phi}_{-\vec{k}}^{+} \\
& -\frac{1}{4 !} \sum_{\vec{k}_{1} \ldots \vec{k}_{4}} \tilde{\mathfrak{M}}_{4}^{(0)} \hat{\phi}_{\vec{k}_{1}}^{+} \hat{\phi}_{\vec{k}_{2}}^{+} \hat{\phi}_{\vec{k}_{3}}^{+} \phi_{\vec{k}_{4}}^{+} \delta_{\vec{k}_{1}+\vec{k}_{2}+\vec{k}_{3}+\vec{k}_{4}}, \\
\bar{M}^{+}= & \tilde{M}^{+}+\tilde{\varepsilon}_{2} \frac{\overline{\mathfrak{M}}_{3}^{(0)}}{\overline{\mathfrak{M}}_{4}^{(0)}}+\frac{1}{3} \frac{\left(\overline{\mathfrak{M}}_{3}^{(0)}\right)^{3}}{\left(\overline{\mathfrak{M}}_{4}^{(0)}\right)^{2}} \\
\bar{\varepsilon}_{2}(k)= & \tilde{\varepsilon}_{2}(k)+\frac{1}{2} \frac{\left(\overline{\mathfrak{M}}_{3}^{(0)}\right)^{2}}{\overline{\mathfrak{M}}_{4}^{(0)}}
\end{aligned}
$$

The chemical potential $\mu_{+}^{(1)}$ is determined from the equation

$$
\frac{\partial \ln \Xi_{1}}{\partial \beta \mu_{+}^{(1)}}=\langle N\rangle
$$

Provided the terms of the fourth order in (4.13) are neglected $\mu_{+}^{(1)}$ is equal to

$$
\mu_{+}^{(1)}=-\frac{1}{2 \beta} \frac{\mathfrak{M}_{2}^{(0)} \tilde{a}(\beta)-\frac{1}{3}\left(\mathfrak{M}_{3}^{(0)}\right)^{3} /\left(\mathfrak{M}_{4}^{(0)}\right)^{2}}{\mathfrak{M}_{2}^{(0)}+\frac{1}{2} \mathfrak{M}_{3}^{(0)} \tilde{a}(\beta)-\frac{1}{2}\left(\mathfrak{M}_{3}^{(0)}\right)^{2} / \mathfrak{M}_{4}^{(0)}}
$$

As a result, we obtain the equation for the gas-liquid spinodal curve

$$
\tilde{a}(\beta)=-2 \frac{\mathfrak{M}_{2}^{(0)}}{\mathfrak{M}_{3}^{(0)}}+\frac{\mathfrak{M}_{3}^{(0)}}{\mathfrak{M}_{4}^{(0)}}
$$

or

$$
\frac{2}{\pi} \int_{0}^{\infty} \frac{x^{2} \sin x \mathrm{~d} x}{x^{3} T^{*}+24 \eta \sin x}=2 \frac{S_{2}(0)}{S_{3}(0)}-\frac{S_{3}(0)}{S_{4}(0)},
$$

where $S_{n}(0)$ is the $n$th structure factor of the one-component hard-sphere system at $k=0$.

The phase diagram of the RPM (in the considered approximation) is shown in figure 1. The curve with the maximum is the gas-liquid spinodal calculated by (4.17).The Percus-Yevick approximation is used for $S_{2}(0)$. The straight line calculated by (4.2)-(4.3) corresponds to the charge ordering phase transition. The gas-liquid critical point is located at $T_{\mathrm{c}}^{*}=0.0502$ and $\eta_{\mathrm{c}}=0.022$. While the value for $T_{\mathrm{c}}^{*}$ is in good agreement with the recent data of computer simulations $[28,29]\left(T_{\mathrm{c}}^{*} \simeq 0.05\right)$, the critical density is underestimated $\left(\eta_{\mathrm{c}} \simeq 0.04\right)$.

\section{Conclusions}

Based on our previous studies, we develop the approach which allows one to obtain the GLW Hamiltonian defined in the phase space of the fluctuating fields $\hat{\phi}_{\vec{k}}$ conjugate to the fluctuating densities connected with the order parameter. We use this approach to the study of both the SBFM and the RPM. In the former case we 


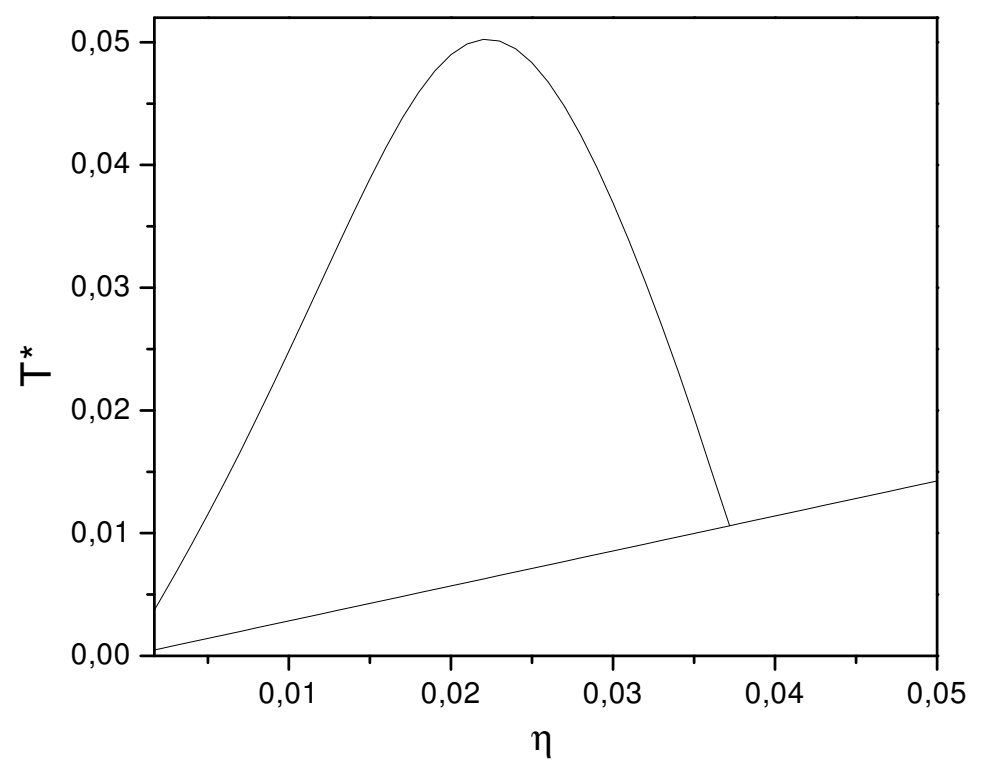

Figure 1. The phase diagram of the RPM (see the text for the explanation).

consider the Gaussian approximation only and show that the equations obtained for the phase instability boundaries are the same as those found in [13] within the framework of the CV method. For the RPM we calculate the phase diagram taking into account the powers of $\hat{\phi}_{\vec{k}}$ higher than the second one. The obtained value for the gas-liquid critical temperature correlates well with the MC simulation data.

The proposed approach can also be used in the case when both long-range (Coulombic) and short-range (i.e., van der Waals) interactions are involved the model simultaneously. This task will be considered elsewhere.

\section{Appendix A}

$$
\begin{aligned}
\hat{D}_{1}(\hat{\phi}) & =\sum_{\vec{k}} \sum_{\gamma=1}^{m} \mathfrak{M}_{1}^{\gamma} \hat{\phi}_{\vec{k}, \gamma}, \\
\hat{D}_{2}(\hat{\phi}) & =\frac{1}{2 !} \sum_{\vec{k}_{1} \vec{k}_{2}} \sum_{\gamma_{1}, \gamma_{2}} \mathfrak{M}_{2}^{\gamma_{1}, \gamma_{2}}\left(k_{1}, k_{2}\right) \phi_{\vec{k}_{1}, \gamma_{1}} \phi_{\vec{k}_{2}, \gamma_{2}}, \\
\hat{D}_{3}(\hat{\phi}) & =\frac{1}{3 !} \sum_{\vec{k}_{1} \vec{k}_{2} \vec{k}_{3}} \sum_{\gamma_{1}, \gamma_{2}, \gamma_{3}} \mathfrak{M}_{3}^{\gamma_{1}, \gamma_{2}, \gamma_{3}}\left(k_{1}, k_{2}, k_{3}\right) \phi_{\vec{k}_{1}, \gamma_{1}} \phi_{\vec{k}_{2}, \gamma_{2}} \phi_{\vec{k}_{3}, \gamma_{3}}, \\
\hat{D}_{4}(\hat{\phi}) & =\frac{1}{4 !} \sum_{\vec{k}_{1} \ldots \vec{k}_{4}} \sum_{\gamma_{1}, \ldots, \gamma_{4}} \mathfrak{M}_{4}^{\gamma_{1}, \ldots, \gamma_{4}}\left(k_{1}, \ldots, k_{4}\right) \phi_{\vec{k}_{1}, \gamma_{1}} \phi_{\vec{k}_{2}, \gamma_{2}} \phi_{\vec{k}_{3}, \gamma_{3}} \phi_{\vec{k}_{4} \gamma_{4}} .
\end{aligned}
$$

Here the $n$th cumulant $\mathfrak{M}_{n}^{\gamma_{1}, \ldots, \gamma_{n}}\left(k_{1}, \ldots, k_{n}\right)$ is connected with $S_{\gamma_{1} \ldots \gamma_{n}}\left(k_{1}, \ldots, k_{n}\right)$, the $n$-particle partial structure factor of the RS, by means of the relation

$$
\mathfrak{M}_{n}^{\gamma_{1} \ldots \gamma_{n}}\left(\vec{k}_{1}, \ldots, \vec{k}_{n}\right)=\sqrt[n]{N_{\gamma_{1}} \ldots N_{\gamma_{n}}} S_{n}^{\gamma_{1} \ldots \gamma_{n}}\left(k_{1}, \ldots, k_{n}\right) \delta_{\vec{k}_{1}+\cdots+\vec{k}_{n}}
$$

$\delta_{\vec{k}_{1}+\cdots+\vec{k}_{n}}$ is a Kronecker symbol. 


\section{Appendix B}

$$
\begin{aligned}
\hat{D}_{3}\left(\chi_{1}, \chi_{2}\right)= & \frac{1}{3 !} \sum_{\vec{k}_{1} \ldots \vec{k}_{3}} \frac{1}{(\sqrt{2})^{3}}\left(\check{\mathfrak{M}}_{3}^{(0)} \chi_{1, \vec{k}_{1}} \chi_{1, \vec{k}_{2}} \chi_{1, \vec{k}_{3}}+3 \check{\mathfrak{M}}_{3}^{(1)} \chi_{1, \vec{k}_{1}} \chi_{1, \vec{k}_{2}} \chi_{2, \vec{k}_{3}}\right. \\
& \left.+3 \check{\mathfrak{M}}_{3}^{(2)} \chi_{1, \vec{k}_{1}} \chi_{2, \vec{k}_{2}} \chi_{2, \vec{k}_{3}}+\check{\mathfrak{M}}_{3}^{(3)} \chi_{2, \vec{k}_{1}} \chi_{2, \vec{k}_{2}} \chi_{2, \vec{k}_{3}}\right) \delta_{\vec{k}_{1}+\vec{k}_{2}+\vec{k}_{3}}
\end{aligned}
$$

where

$$
\begin{aligned}
\check{\mathfrak{M}}_{3}^{(0)}= & \mathfrak{M}_{3}^{(0)} \mathcal{A}^{3}+3 \mathfrak{M}_{3}^{(1)} \mathcal{A}^{2} \mathcal{C}+3 \mathfrak{M}_{3}^{(2)} \mathcal{A} \mathcal{C}^{2}+\mathfrak{M}_{3}^{(3)} \mathcal{C}^{3} \\
\check{\mathfrak{M}}_{3}^{(1)}= & \mathfrak{M}_{3}^{(0)} \mathcal{A}^{2} \mathcal{B}+\mathfrak{M}_{3}^{(1)} \mathcal{A}(\mathcal{A D}+2 \mathcal{B C})+\mathfrak{M}_{3}^{(2)} \mathcal{C}\left(2 \mathcal{A D}+\mathcal{B C}+\mathfrak{M}_{3}^{(3)} \mathcal{C}^{2} \mathcal{D}\right. \\
\check{\mathfrak{M}}_{3}^{(2)}= & \mathfrak{M}_{3}^{(0)} \mathcal{A} \mathcal{B}^{2}+\mathfrak{M}_{3}^{(1)} \mathcal{B}(2 \mathcal{A D}+\mathcal{B C})+\mathfrak{M}_{3}^{(2)} \mathcal{D}\left(\mathcal{A D}+2 \mathcal{B C}+\mathfrak{M}_{3}^{(3)} \mathcal{C} \mathcal{D}^{2}\right. \\
\check{\mathfrak{M}}_{3}^{(3)}= & \mathfrak{M}_{3}^{(0)} \mathcal{B}^{3}+3 \mathfrak{M}_{3}^{(1)} \mathcal{B}^{2} \mathcal{D}+3 \mathfrak{M}_{3}^{(2)} \mathcal{B} \mathcal{D}^{2}+\mathfrak{M}_{3}^{(3)} \mathcal{D}^{3} \\
\hat{D}_{4}\left(\chi_{1}, \chi_{2}\right)= & \frac{1}{4 !} \sum_{\vec{k}_{1} \ldots \vec{k}_{4}} \frac{1}{(\sqrt{2})^{4}}\left(\check{\mathfrak{M}}_{4}^{(0)} \chi_{1, \vec{k}_{1}} \chi_{1, \vec{k}_{2}} \chi_{1, \vec{k}_{3}} \chi_{1, \vec{k}_{4}}\right. \\
& \quad+4 \check{\mathfrak{M}}_{4}^{(1)} \chi_{1, \vec{k}_{1}} \chi_{1, \vec{k}_{2}} \chi_{1, \vec{k}_{3}} \chi_{2, \vec{k}_{4}}+6 \check{\mathfrak{M}}_{4}^{(2)} \chi_{1, \vec{k}_{1}} \chi_{1, \vec{k}_{2}} \chi_{2, \vec{k}_{3}} \chi_{2, \vec{k}_{4}} \\
& \left.\quad+4 \check{\mathfrak{M}}_{4}^{(3)} \chi_{1, \vec{k}_{1}} \chi_{2, \vec{k}_{2}} \chi_{2, \vec{k}_{3}} \chi_{2, \vec{k}_{4}}+\check{\mathfrak{M}}_{4}^{(4)} \chi_{2, \vec{k}_{1}} \chi_{2, \vec{k}_{2}} \chi_{2, \vec{k}_{3}} \chi_{2, \vec{k}_{4}}\right) \delta_{\vec{k}_{1}+\vec{k}_{2}+\vec{k}_{3}+\vec{k}_{4}},
\end{aligned}
$$

where

$$
\begin{aligned}
\check{\mathfrak{M}}_{4}^{(0)}= & \mathfrak{M}_{4}^{(0)} \mathcal{A}^{4}+4 \mathfrak{M}_{4}^{(1)} \mathcal{A}^{3} \mathcal{C}+6 \mathfrak{M}_{4}^{(2)} \mathcal{A}^{2} \mathcal{C}^{2}+4 \mathfrak{M}_{4}^{(3)} \mathcal{A} \mathcal{C}^{3}+\mathfrak{M}_{4}^{(4)} \mathcal{C}^{4} \\
\check{\mathfrak{M}}_{4}^{(1)}= & \mathfrak{M}_{4}^{(0)} \mathcal{A}^{3} \mathcal{B}+\mathfrak{M}_{4}^{(1)} \mathcal{A}^{2}(\mathcal{A D}+3 \mathcal{B C})+3 \mathfrak{M}_{4}^{(2)} \mathcal{A C}(\mathcal{A D}+\mathcal{B C}) \\
& +\mathfrak{M}_{4}^{(3)} \mathcal{C}^{2}\left(3 \mathcal{A D}+\mathcal{B C}+\mathfrak{M}_{4}^{(4)} \mathcal{C}^{3} \mathcal{D}\right. \\
\check{\mathfrak{M}}_{4}^{(2)}= & \mathfrak{M}_{4}^{(0)} \mathcal{A}^{2} \mathcal{B}^{2}+2 \mathfrak{M}_{4}^{(1)} \mathcal{A B}(\mathcal{A D}+\mathcal{B C})+\mathfrak{M}_{4}^{(2)}\left(\mathcal{A}^{2} \mathcal{D}^{2}+\mathcal{B}^{2} \mathcal{C}^{2}+4 \mathcal{A B C}\right) \\
& +2 \mathfrak{M}_{4}^{(3)} \mathcal{C D}(\mathcal{A D}+\mathcal{B C})+\mathfrak{M}_{4}^{(4)} \mathcal{C}^{2} \mathcal{D}^{2} \\
\check{\mathfrak{M}}_{4}^{(3)}= & \mathfrak{M}_{4}^{(0)} \mathcal{A} \mathcal{B}^{3}+\mathfrak{M}_{4}^{(1)} \mathcal{B}^{2}\left(3 \mathcal{A D}+\mathcal{B C}^{(2)}+3 \mathfrak{M}_{4}^{(2)} \mathcal{B D}(\mathcal{A D}+\mathcal{B C})\right. \\
& +\mathfrak{M}_{4}^{(3)} \mathcal{D}^{2}(\mathcal{A D}+3 \mathcal{B})+\mathfrak{M}_{4}^{(4)} \mathcal{C} \mathcal{D}^{3} \\
\check{\mathfrak{M}}_{4}^{(4)}= & \mathfrak{M}_{4}^{(0)} \mathcal{B}^{4}+4 \mathfrak{M}_{4}^{(1)} \mathcal{B}^{3} \mathcal{D}+6 \mathfrak{M}_{4}^{(2)} \mathcal{B}^{2} \mathcal{D}^{2}+4 \mathfrak{M}_{4}^{(3)} \mathcal{B D} \mathcal{D}^{3}+\mathfrak{M}_{4}^{(4)} \mathcal{D}^{4}
\end{aligned}
$$

\section{References}

1. Chen X.S., Forstmann F. // J. Chem. Phys., 1992, vol. 97, p. 3696.

2. Ciach A., Stell G. // J Mol. Liq., 2002, vol. 87, p. 53.

3. Brilliantov N.V., Valleau J.P. // J. Chem. Phys., 1998, vol. 108, p. 1115.

4. Brilliantov N.V. // Phys. Rev. E, 1998, vol. 58, p. 2678.

5. Brilliantov N.V., Loskutov A.Yu., Malinin V.V. // Theoret. and Math. Phys., 2002, vol. 130, p. 145 (in Russian).

6. Yukhnovskii I.R. Phase Transitions of the Second Order: Collective Variables Method. Singapore, World Scientific, 1987. 
7. Yukhnovskii I.R. // Physica A, 1990, vol. 168, p. 999.

8. Yukhnovskii I.R. // Proceed. of the Steclov Inst. of Math., 1992, vol. 2, p. 223.

9. Yukhnovskii I.R., Idzyk I.M., Kolomietc V.O. // J. Stat. Phys., 1995, vol. 80, p. 405.

10. Zubarev D.N. // DAN USSR, 1964, vol. 95, p. 757 (in Russian).

11. Yukhnovskii I.R., Holovko M.F. Statistical Theory of Classical Equilibrium Systems. Kiev, Naukova Dumka, 1980 (in Russian).

12. Patsagan O.V., Yukhnovskii I.R. // Theoret. Math. Phys., 1990, vol. 83, p. 387.

13. Yukhnovskii I.R., Patsahan O.V.// J. Stat. Phys., 1995, vol. 81, p. 647.

14. Patsahan O.V., Kozlovskii M.P., Melnyk R.S. // J. Phys.: Condens. Matter, 2000, vol. 12, p. 1595.

15. Patsahan O.V., Patsahan T.M. // J. Stat. Phys., 2001, vol. 105, p. 287.

16. Patsahan O.V.// Physica A, 1999, vol. 272, p. 358.

17. Patsahan O.V., Melnyk R.S., Kozlovskii M.P. // Condens. Matter Phys., 2001, vol. 4, p. 235.

18. Hubburd J., Schofield P. // Phys. Lett. A, 1972, vol. 40, p. 245.

19. Patsahan O.V., Holovko M.F. On the theory of phase transitions of fluids in porous media. Preprint of the Institute for Condensed Matter Physics, ICMP-01-08U, Lviv, 2001, 24 p. (in Ukrainian).

20. Hubburd J. // Phys. Rev. Lett., 1959, vol 3, p. 77; Stratonovich R.L. // Sov. Phys. Dokl., 1957, vol. 2, p. 416.

21. Kubo R. // J. Phys. Soc. Jpn., 1962, vol. 17, p. 100.

22. Shiryajev A.N. Probability. Moscow, Nauka, 1989 (in Russian).

23. Patsahan O.V. // Ukr. Fiz. Zh., 1996, vol. 41, p. 877 (in Ukrainian).

24. Stell G. The Equilibrium Theory of Classical Fluids. New York, Benjamin, 1964.

25. Patsahan O.V. // Condens. Matter Phys., 1995, No. 5, p. 124.

26. Kozlovskii M.P., Patsahan O.V., Melnyk R.S. // Ukr. Fiz. Zh., 2000, vol. 45, p. 381 (in Ukrainian).

27. Weeks J.D., Chandler D., Andersen H.C. // J. Chem. Phys., 1971, vol. 54, p. 5237.

28. Orkoulas G., Panagiotopoulos A.Z. // J. Chem. Phys., 1999, vol. 110, p. 1581.

29. Caillol J.-M., Levesque D., Weis J.-J. Critical behavior of the restricted primitive model revisited. Preprint cond-mat/0201301 v1. 


\section{Гамільтоніан Гінзбурга-Ландау-Вільсона для багатокомпонентних неперервних систем: мікроскопічний підхід}

\section{О.В.Пацаган}

Інститут фізики конденсованих систем НАН України, 79011 Львів, вул. Свєнціцького, 1

Отримано 13 квітня 2002 р.

Недавно ми запропонували мікроскопічний підхід до опису фазових переходів і критичних явищ в бінарних флюїдних сумішах, який базується на методі колективних змінних (КЗ) з виділеною системою відліку. Цей підхід дозволив нам отримати функціонал гамільтоніана Гінзбурга-Ландау-Вільсона (ГЛВ), який виражений в термінах колективних змінних ("густинних" змінних). Відповідний набір колективних змінних включав змінну, пов' язану з параметром порядку. В цій статті, базуючись на попередніх результатах, ми будуємо гамільтоніан ГЛВ у фазовому просторі "польових" змінних $\hat{\phi}_{\vec{k}}$ (флуктуюючих полів), спряжених до "густинних" змінних. Ми застосовуємо отриманий функціонал ГЛВ до вивчення бінарної симетричної суміші і найпростішої іонної моделі. В першому випадку ми розглядаємо тільки гауссове наближення і показуємо, що отримані результати є такі ж як і отримані раніше в рамках методу КЗ. В другому випадку ми обчислюємо фазову діаграму, враховуючи вищі степені $\hat{\phi}_{\vec{k}}$ ніж друга.

Ключові слова: фазовий перехід, двокомпонентна неперервна система, параметр порядку, флуктуююче поле

PACS: $05.70 . F h, 05.70 . J k, 65.10 .+h$ 